\title{
ENERGY VARIATIONS IN DIFFUSIVE CAVITY GRONTH
}

by James $R$. Rice and Tze-jer Chuang*

Division of Engineering, Brown University, Providence, R. I. 02912, USA

\section{June 1979}

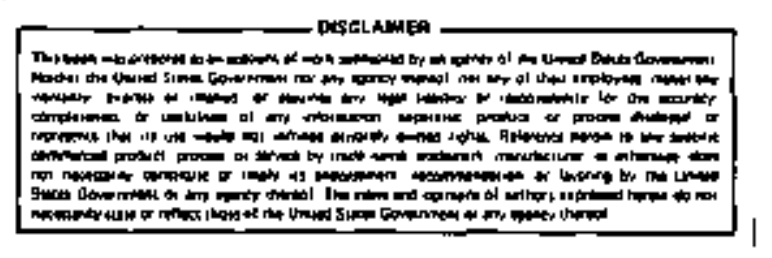

* Current Affiliation: Advanced Systems Technology, Westinghouse Electric Corporation, Pittsburgh, PA 15221, USA 


\section{DISCLAIMER}

This report was prepared as an account of work sponsored by an agency of the United States Government. Neither the United States Government nor any agency Thereot, nor any of their employees, makes any warranty, express or implied, or assumes any legal liability or responsibility for the accuracy, completeness, or usefulness of any information, apparatus, product, or process disclosed, or represents that its use would not infringe privately owned rights. Reference herein to any specific commercial product, process, or service by trade name, trademark, manufacturer, or otherwise does not necessarily constitute or imply its endorsement, recommendation, or favoring by the United States Government or any agency thereof. The views and opinions of authors expressed herein do not necessarlly state or reflect those of the United States Government or any agency thereof. 


\section{DISCLAIMER}

Portions of this document may be illegible in electronic image products. Images are produced from the best available original document. 
ABSTRACT

We discuss the assigntent of boundary values for the cherical potential and the calculation of encrgy release rates for the growth of creep cavities along grain boundaries by self-diffusion. For simplicity it is assumed that the boundaries are flat and that surface and grain-boundary diffusion are the doninant transport nechanisms. As matter diffuses fron the void surface into and along the grain boundary, misfit residual stresses are induced to alleviate the high stress concentration ahead of the cavity apex. As a result, it is shown that the contribution of strain energy terms to the chenical potential can be neglected in typical cases. Also, contrary to the Griffith crack extension nodel, the energy dissipation incurred by diffusive removal of naterial from the cavity surface and deposition in the grain boundary is a major term in the energy transfers associated with cavity growth. We show that the primary energy "sink" in diffusive cavity growth arises fron the work done by the grain-boundary nornal stress when matter is inserted in the near-tip region by diffusion, and not from the loss of strain energy of natter that is removed from the cavity at its tip or from a work of bond separation. We also comment on therrodynamic restrictions on the angle formed by the void surfaces at their apex, where they join the grain boundary. Further, our derivation of boundary values for the chemical potential is carried out in a manner appropriate for arbitrarily large but elastic distortions of naterial ņear the cavity tip and, by contrast to most previous work in the area, we include rigorously the effects of surface tension (i.e., of "surface stress", as distinct from surface energy). 


\section{INTRODUCTION}

When subject to creep conditions, polycrystalline materials tend to develop cavities at grain interfaces. Their growth and final coalescence leads to intergranular failure. The kinetics of cavity growth by diffusion has becone a topic of extensive recent interest, and there is not full agreenent in the literature on fundamentals of the subject. The papers by Hull and Rimmer (1959) and others (Speight and Harris, 1967; Weertman, 1973; Vitovec, 1972; Raj and Ashby, 1975) considered the diffusive growth of an array of spherical-caps (or lenticularl cavities in a planar grain interface. This assunption as to cavity shape implies that surface diffusion is sufficiently rapid that cavity growth is controlled only by grain boundary (g.b.) diffusion. Further, the grains were assumed to be non-deforming (rigid), and the result predicted is that the growth rate varies linearly with the applied stress. However, conditions do not always allow this quasi-equilibrium cavity shape and g.b. cavities sometines have an elongated, crack-like shape. Hence, Chuang and Rice (1973) and others (Vitek, 1978; Speight et al, 1978; Beere and Speight, 1978; Chuang et al, 1979) considered the other limiting case of a thin crack-1ike cavity growing in a g.b. and established different relationships between applied stress and growth speed.

In an overview of non-equilibriun madels, Chuang et al. (1979) examined the entire spectrun of interfacial void shapes in diffusive cavitation and concluded that over a variety of conditions, a cavity can grow from one extreme case of slow advance in a lenticular shape to the other extreme case of rapid advance in a crack-ljke shape. The growth 
mechanisms on which those analyses are based are such that under the action of the applied stress normal to the boundary where cavities are located, natter on the cavity surfaces is driven by surface diffusion toward the cavity apex and into and along the g.b.

Based on this kinetic model, it is worthwhile to investigate the thermodynamic forces driving a cavity. Indeed, the subject of chemical forces in a stressed body containing defects has been studied by several authors. Specifically, Stevens and Dutton (1971) considered Griffith crack propagation by mass transport and formulated the thermodynamic potentials for increnental advance of crack due to various diffusion paths. McCartney (1977) and Heald and Speight (1977) investigated the thernodynamic stability of a cavity and determined the shape at which subcritical growth can be maintained. Their studies are essentially linited to cases in which cavities are lacated in a perfect crystal. However, it is well recognized that grain boundaries are perfect matter sinks (or vacancy sources) and the fact that voids are often observed at the g.b. suggests that it plays an important role during the stages of nucleation and growth.

The objective of this paper is to re-examine the formulation of chenical potentials and the calculation of energy release rates in cavity growth. We place emphasis on the special features induced by the existence of grain boundaries. Their action as matter sinks can change the whole picture of the formulation. Specifically, in contrast to Griffith cracks, we show that the strain energy term in the chenical potential expression can typically be neglected even in cavity growth in a relatively narrow crack-like shape. We also show that the najor sink 
of energy in cavity growth is fron the work of normal stresses on the effective opening of the grain boundary due to the addition of natter to it by diffusion.

While our considerations have modelled the material as an elastic solid, undergoing self-diffusion along interfaces, it is well to remenber that the cavitation process is often accompanied by significant anounts of plastic creep flow through dislocation motion. 
II. THE BQUNDARY VALUES OF CHEMICAL POTENTIAL

In phenomenological terms, diffusive fluxes of a substance are driven by thermodynamic forces generated by chemical potential gradients. Consider a stressed body containing defects in the form of voids and grain boundaries, and suppose for simplicity that the body consists of a single component which, again for simplicity, is taken as isotropic with respect to its elastic and surface energy properties. Temperature is held constant and we suppose that an unstressed matter reservoir, without defects, is available at the same temperature as the body, All thermodynamic potentials, including the chemical potential $W$, can arbitrarily be assigned the value zero in the reservoir. It is conventional to define $w$ in units of energy per aton although the concepts are continum in character and treat matter as being indefinitely divisible; atoms are mèrely a convenient unit for mass.

The classic work on establishing boundary values for $\mu$ is that of Herring $(1951,1953)$. We follow essentially his procedures, but three comments are in order. First, since we shall assume that natter is always added coherently to surfaces, at full composition equilibrium with the inaediately adjoining bulk, there is no need to distinguish separate potentials for atoms and vacancies as he does. Indeed, Herring makes similar assumptions about matter addition, so that in the end the distinction is unnecessary and only $\mu_{a}-\mu_{v}$ appears in his formulae ("a" denotes atons, " $v^{\text {tr }}$ vacancies). Second, although Herring classifies sarefully the difference between surface energies and surface tensions (i.e., surface stresses), in a context which assunes clearly that surface energies are deperdent on elastic strains of surface elements, in his evaluations of 
he gives no discussion of energy changes that come about because matter addition will, in general, alter the strains along a surface. We derive expressions for $\downarrow$ in a manner appropriate to arbitrarily large elastic distortions (important because some models for diffusive cavitation assume large strains at the cavity tip) and show, by a simple verbal argtment in the following text and a more detalled analysis in the Appendix, that Herring's expressions do in fact renain valid so long as the terms within then are properly referenced to the current deformed state. Third, although some of Herring's discussion of potentials is directed to (global) non-equilibriwn states, notably to diffusion, his methods of evaluating $\mu$ are phrased mostly in terms of conditions for equilibriun. Our methods are somewhat nore in keping with the spirit of irreversible thermodynamics although, certainly, we adopt tacitly the notions of local thermodynamic equilibrium which are inherent to the accepted, if thereby limited, procedures of that subject.

To proceed, we observe that $p$ is defined at any location within the stressed body such that $\mu \delta_{n}$ is the reversible work of taking an infinitesimal element of natter, equivalent to $\delta$ n atoms, from the reservoir and placing it at that location, while the displacenents are held fixed on the loaded external boundary. Equivalently, $4 \delta \mathrm{n}=6 \mathrm{~F}$ where $F$ is the total Helnholtz free energy of the body, and nore generally, when matter is transferred without restrictions on the external boundary displacenents,

$$
\delta F=\delta w+\mu \delta n
$$

where $\delta w$ is the work of external loads. Our concern here is with 
matter addition to (or removal from) void surfaces and grain boundaries. If these, collectively, have an area in the current deformed configuration denoted by $A$, then $W$ satisfies

$$
\delta F=\delta W+\int_{A} \omega \delta \mathrm{dA}
$$

for arbitrary additions of infinitesinal matter layers to $A$, consisting of $t \mathrm{~N}$ atoms per unit (current) area. Expressions in the form of (2) arise in "internal variable" formulations (Rice, 1974) of inelastic processes in solids, due to structural rearrangements of constituent elements of material by diffusion or (with appropriate terms analogous to $p d N$ l stip, phase changes, etc. In applying (2) we will generally write $\delta N=\delta t / \Omega$ where $\delta$ is the local thickness of the (coherent2y) added layer and $\Omega$ is the local volune per atom in the deformed configuration. Further, $F$ will be represented as $F_{e}+F_{s}$, where $F_{e}$ is the elastic strain energy, of local density $w$ per unit volume of the current deformed configuration, and $F_{s}$ is the energy of interfaces, of density $\gamma$ per unit (current) area $\left(Y=Y_{s}\right.$ on cavity surfaces and $Y=\gamma_{b}$ on grain boundaries).

Consider the addition of a layer of thatter of (variable) local thickness $6 t$ over some portion of a cavity surface, under circumstances for which the external boundary is fixed $(\sigma W=0)$. To obtain $\omega$ we need to calculate $\delta F$, and this has contributions from the following sources: (i) the added layer must be given a strain energy $w$ to make it fit coherently; (ii) the area of the surface changes such that, if additional 
elastic strains $\delta \varepsilon_{\alpha B}$ of surface elements are neglected, $\delta(d A)=$ $-\left(k_{1}+k_{2}\right) \xi t d A$ by an easy geometric construction, where $\kappa_{1}$ and $k_{2}$ are the principal curvatures and the sign convention is such that both would be positive on a spherical cavity; (iii) surface elements strain by $\delta_{\alpha \beta}$ causing a change in $Y$ and an additional change in $d A$; (iv) the tractions acting on the bulk solid inmediately below the surface, which are in general non-zero whenever surface tensions exist, are carried through sone additional displacements $\delta u_{i}$ when matter is added, thus causing a change in strain energy of naterial external to the surface. Herring's $(1951,1953)$ discussion makes no reference to (iii) and (iv). It is carried out as if the product $Y$ dA is unaffected by the strains $6 \mathrm{e}_{a \beta} ;$ in such circunstances the contribution from (iii) would be zero and because (see Appendix) the surface tensions vanish in these circumstances, there would then be no contributions from (iv) either. (Rice and Drucker (1967) observed that when matter is added or removed from an unstressed surface the energy alteration analogous to (iv) is of second order in \&t, hence zero for our present purposes).

We evaluate of as if $\gamma$ dA was invariant to strain and then explain briefly (relegating a detailed analysis to the Appendix) why the result is valid in general. Hence the contributions to $\delta F$ arise from (i) and (ii), and are

$$
\dot{\delta} F=\int_{A} w \delta t d A+\int_{A} Y_{S}\left[-\left(\alpha_{1}+\kappa_{2}\right) \delta t\right] d A .
$$

If we now write $\delta t=\Omega \delta \mathrm{N}$ and compare with $(1),-$ it is seen that

$$
w=w \Omega-Y_{5}\left(\kappa_{1}+\kappa_{2}\right) \Omega
$$


on a cavity surface. This is the same as the expression given by Herring, so long as it is understood that $w, \Omega, \gamma_{s}$ and the $x^{\prime} s$ are referenced to the current deformed state at the place of matter addition.

Now, let us drop the generally untenable assumption that $Y$ dA is invariant to surface strain and that surface tensions vanish. Ne can calculate $\delta \mathrm{F}$ by first carrying out steps (i) and (ii), while applying to the system whatever system of (workless) forces are necessary to prevent occurrence of the surface strains $\delta \varepsilon_{a \beta}$ and displacements $\delta u_{i}$. This gives a of exactly as in eq. (3) and the net additional $6 \mathrm{~F}$, as sociated with steps (iii) and (iv), is just minus the work of removing this additional set of forces. But the final state to which the system goes is one of elastic (as opposed to compositional, or surface shape) equilibrium, and at this state $F$ is stationary with respect to elastic distortions. Hence the net work of removing the set of constraining forces is a quantity of second order in $\delta t$, and the net contribution of (iii) and (iv) to $\delta F$ is therefore zero to the first order terns of interest. Hence (3) and, particularly, (4) for $\mu$ are valid generally. A fuller analysis, necessarily of sone mathematical complexity, is given in the Appendix. Indeed, it is shown there that when surface tensions exist $\delta F_{e}$ and $\delta F_{s}$ both separately contain terals of first order in ot (in addition to those already listed in eq. 3), but these terms are of opposite sign and cancel when $\delta F=\delta F_{e}+\delta F_{5}$ is formed.

often, throughout the body of the paper, we simplify derivations by neglecting surface tension and the variation of $\gamma$ dA with surface strain. As we remark, the final results in each case are valid without these assumptions, provided that terms are properly interpreted, and details 
of complete derivations are given in the Appendix.

Consider now the addition of a matter layer $6 t$ to a flat grait boundary subject to tensile stress, again under conditions for which $\delta W=0$. The layer must be given strain energy appropriate for coherent fit, and the boundary must be separated by the distance to to allow the new natter, resulting in a change $-\int 0 \delta t d A$ of strain energy of the adjoining naterial, where $\sigma$ is the normal stress ("true" stress, i.e, force per unit current areal acting on the grain boundary. Thus

$$
\delta F=\int_{A}(w-\sigma) d t d A
$$

and writing ot $=\Omega \delta \mathrm{N}$,

$$
\mu=w-\sigma \Omega
$$

glong a grain boundary. Again, this agrees with Herring's result 80 long as the terns are proporly referenced to the current deformed state, and (see Appendix) the result remains valid when surface tension along the grain boundary and dependence of $\gamma_{b}$ on strain is included.

Indeed, this last formula nakes evident our reasons for care in defining quantities per unit volume or area of the deformed configuration. The difference between of and on $\Omega_{r}$, where $a_{r}$ is the value of $\Omega$ in the unstressed reservoir, is itself of the same oroer as the strain energy term wh in (6). If, instead $\hat{w}$ is the strain energy per unit volune as measured in the unstressed state, and $\hat{\sigma}$ and $\vec{r}_{s}$ are force and surface energy per unit area of the unstressed state (so that $\vec{j}$ is a 
"nominal" stress), then it is straightforward to show that (4) and (6) are modified to

$$
\mu=\hat{w}_{r}-\hat{Y}_{s}\left(1+\varepsilon_{n}\right)\left(\kappa_{1}+\kappa_{2}\right) \Omega_{r}
$$

on the cavity surface and

$$
\mu=\hat{w}_{r}=\hat{o}\left(1+\varepsilon_{n}\right) \Omega_{r} .
$$

on the grain boundary, where $\varepsilon_{n}$ is the strain of adjoining material elenents in the direction normal to the surface being considered. Obviously, $\hat{o}_{n}$ is of the sane order as $\dot{w}$, and hence great care is required in discussing the effect of strain energy contributions to the chenical potentials (Stevens and Dutton, 1971; McCartney, 1977; Charles, 1976; Dutton and Puls, 1978). Similar remarks apply to $\left(4^{\prime}\right)$ since $\kappa_{1}{ }^{\prime}$ is generally of the same order as $\sigma$ at a cavity tip (see below).

In a material capable of matter transport by diffusion, $u$ must be continuous at the cavity apex where it neets the grain boundary; otherwise an unbounded matter flux would result there. Hence, when the strain energy terns are negligible

$$
Y_{5}\left(\kappa_{10}+\kappa_{20}\right)=\sigma_{0}
$$

where $\kappa_{10}, \kappa_{20}$ and $\sigma_{0}$ are, respectively, the cavity surface curvatures as the apex is approached along the cavity walls and the grain boundary normal stress as the apex is approached. Using this formula as an approximate 
estimate of stress in the vicinity of the apex, we can estimate the relative importance of the strain energy terms. For simplicity, consider cylindrical voids under plane strain conditions $\left(\kappa_{1}=\kappa_{,} \kappa_{2}=0\right)$ and assume linear elasticity. Then

$$
W \approx \frac{1-v^{2}}{2 \mathrm{E}} \quad \sigma_{0}^{2} \approx \frac{\gamma_{\mathrm{s}} K_{0}}{2 \mathrm{E}} \gamma_{\mathrm{s}} \mathrm{K}_{0} \approx \frac{\mathrm{b}}{500_{0}} \gamma_{\mathrm{s}} \kappa_{0}
$$

where $b$ is the lattice spacing, $p_{0}=1 / \mathrm{k}_{0}$ is the radius of curvature of the cavity wall at the apex, and we use the estimate $\gamma_{S} \approx E b / 25$. Since $o_{a}$ typically has values of 50 to $500 \mathrm{~b}(i . e, 100 \AA$ to $1 \mathrm{\mu m}\}$, the strain energy term is quite negligible.

We enphasize, however, that this result occurs because of the mobility of natter in the circunstances considered, Surface diffusion allows the attainment of large values of $p_{p}$ (coupared, say, to that for a flat. Griffith crack\} and misfit stresses induced by matter flow into the $\mathrm{g} . \mathrm{b}$. effectively thicken the boundary near the tip, alleviating the stress concentration normally associated with cracks or cavities. Very different conclusions on the importance of $w$ could arise when the cavity does not lie along, or itself constitute, a high diffusivity path.

These latter circumstances seem to coincide with those assumed by Stevens and Dutton (1971) and Dutton and Puls (1978) for the diffusive growth of Griffith cracks and, at this point, it is perhaps interesting to compare the directions of matter flow in the different toodels proposed. As Dutton and coworkers renarked, in the case of their Griffith crack extension model the chemical potential at the tip region is very high due to high strain energy density developed in this region, and they consider 
the first term in (4) to dominate. Hence they assuned that the direction of diffusion is away from the tip along the cavity surface. On the other hand, as envisioned in our model, based on the Hull-Rimmer model and its generalizations, misfit stress has been induced to reduce greatly the stress concentration associated with a notch so that the second term of (4) takes over. The chemical potential at the cavity surface far away from the tip is higher than at the tip reglon and the flow is then reversed toward the tip and into the g.b. Intuitively, this must be true since in our model the g.b. which lies ahead of the cavity tip is capable of accepting the natter previously diffused from the cavity surface, whereas in the Griffith model, the cavity is regarded as bejng essentially the same as if it were located in a perfect crystal so that no sinks are available to accomodate the matter, and thus flow must carry atoms away from the tip. However, it remains an open question as to whether a full solution to the coupled equations of elasticity and diffusion would actually produce cavizy growth in the narrow crack-like mode assumed in the Griffith nodel of Dutzon and coworkers. Instegd, e.g., it is possible that a full analysis would merely predict a rounding out of the crack near its tip. By contrast, a variety of complete solutions have been carried out for the model which we envision for cavities along grain boundaries, and these do indeed predict growth, ultimately in a comparatively narrow crack-like mode (Chuang, 1974; Vitek, 1978; Chuang et al., 1979), but with negligible strain energy teras at the tip so long as $\mathfrak{b}_{0} \gg b$. 


\section{ENERGY RELEASE RATE IN CAVITY GROWTH}

In fracture mechanics terminology, there is an energy release rate associated with cavity growth. Since there is not full agreement in the published literature, we examine here the conputation of this rate and answer the question as to where the energy goes for diffusive crack growth. This is done with reference to a plane strain mode (i,e, cylindrical cavities or cracks) so that all works and energies are on a unit thickness basis.

In the case of elastic-brittle crack growth without diffusion, as formulated within the Griffith context, an energy release rate $G$ is defined by

$$
\mathrm{Ga}=\dot{H}_{-} \dot{\mathrm{F}}_{\mathrm{e}}
$$

where a is crack length. Here it is customary to neglect surface tension effects (whether they can be included consistently within a model for a mathesatically sharp tipped erack remains an open question), so that $F_{\text {e }}$ is just the ordinary strain energy of the cracked body as computed from continum elasticity theory and $G$ is given by the well-known expression $\left(1-v^{2}\right) k^{2} / E$ for an isotropic linear elastic solid. Further, in the Griffith nodel dF $\mathrm{dis}_{s}$ written as $\left(2 \gamma_{s}-\gamma_{b}\right)$ da because in an increment da of growth a length $2 \mathrm{da}$ of crack surface is created at the expense of length da of grain boundary. Actually, this should be written as $\left(2 \hat{\gamma}_{s}=\hat{\gamma}_{b}\right) d \hat{a}$, where $\hat{d a}$ is the growth increment as measured off relative to some reference configuration, and the $\hat{r}$ 's are based on unit area of that configuration (the $\hat{\gamma}$ 's are independent of surface strain when 
surface tensions vanish). Understanding the terms in this way one has, for the Griffith model of reversible crack growth

$$
d H=d F=d F_{e}+d F_{s}=d F_{e}+\left(2 Y_{s}-Y_{b}\right) d a
$$

during growth, and hence the required value of $G$ is

$$
G=2 \gamma_{s}-\gamma_{b}
$$

It has been emphasized recently (Rice, 1978) however, that the actual requirenent of thernodynamics for quasi-static, isothermal crack growth should be phrased as the inequality $\dot{H} \geqslant \dot{F}$ (i.e., the inequality assuring non-negative entropy production] which requires only that

$$
\dot{H}-\dot{F} \equiv\left[G-\left(2 Y_{S}-\gamma_{b}\right)\right] \dot{a} \geqslant 0
$$

for growth (or healing, $\dot{a}<0$ ), and this is general enough to include lattice trapping with thernally activated growth at values of $G$ different from (11). Here $2 \gamma_{s}-\gamma_{b}$ can be interpreted as the reversible work of pulling the interface apart against cohesive forces.

The formulation is rather different. for the diffusive growth process considered here, because there is no direct rupturing of cohesive bonds. We assume, consistently with procedures in the thernodynamics of diffusion, that $\psi$ is defined as in eqs. (4) and (6) during processes, and that diffusive fluxes $J$ are established on the cavity surface and grain boundary, such that $J a \mu / \partial s<0$ where $s$ is arc length along the flow path. 
We consider a half-void of length a as in fig. 1 and let $r_{b}$ denote the grain interface and $r_{s}$ the upper surface of the cavity. Then eq. (2), which is regarded as an identity given the definitions of $\mu$, can be written with $F=F_{e}+F_{s}$, where

$$
F_{s}=2 \int_{\Gamma_{s}} Y_{s} d s+\int_{\Gamma_{b}} \gamma_{b} d s
$$

and ds denotes arc length. Hence if an energy release rate is defined as $\dot{W}-\dot{F}_{e}$ the rate is given by

$$
\dot{H}_{-} \dot{\mathrm{F}}_{e}=\dot{\mathrm{f}}_{s}-2 \int_{\Gamma_{s}} \mu \dot{N} \mathrm{ds}-\int_{\Gamma_{b}} \mu \dot{N} \mathrm{ds}
$$

Sone rearrangenents of this equation are useful. We note, however, that contrary to the case for Griffith cracks, the quantity like $G$, namely $\left(\dot{\psi}-\dot{F}_{e}\right) / \dot{a}$, will not in general be simply a function of applied load and notch size but, as show by examples later, will depend on details of the natter placenent along the grain boundary. First, observing that J and $\$$ are related by mass conservation,

$$
\partial J / \partial s+\dot{N}=0,
$$

that $u_{s}=u_{b}, 2 J_{s}=J_{b}$ at the cavity apex, and that $J$ vanishes at the left and right boundaries (the latter if the right boundary is a half-spacing between cavities), integration by parts leads to

$$
\dot{H}-\dot{F}_{e}=\dot{F}_{s}+\left[2 \int_{\Gamma_{s}}\left(-J \partial_{w} / \partial s\right) d s+\int_{\Gamma_{b}}\left(-J \partial_{\mu} / \partial s\right) d s\right] .
$$


Hence the energy release rate $\dot{W}-\dot{F}_{e}$ supplies two terns, the first $\left(\dot{F}_{s}\right)$ being the energy which goes to cavity surface creation and grain boundary removal, and the second being diffusive dissipation in the form of necessarily positive products ( $-\mathrm{J}$ âu/zs).

Next, we observe that directly from elasticity theory, the rate of change in strain energy (calculated, e.g., as by Rice and Drucker (1967), Rice (1968a), and Eshelby (1970) when material is added or removed from surfaces) is

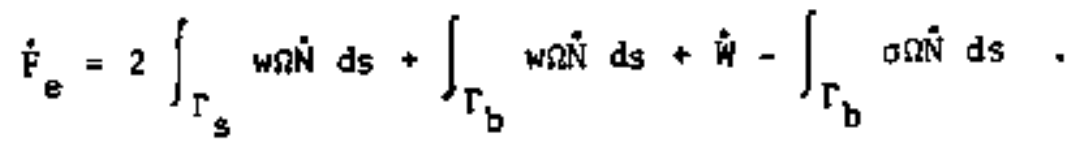

Here si (generally positive on $r_{b}$ and negative on $r_{s}$ ) is the volumetric rate of matter addition per unit area, or rate of thickening $\dot{i}$; the first two terms represent the strain energy of the added matter, and the last two the work of all applied tractions, both external and those (-o) acting along the g.b. Equation (17) is written for the case when no surface tensions exist, consistently with the references by Rice and others cited above. The full forn of the equation is given in the Appendix. Ne can also compute $\dot{F}_{S}$, where $F_{s}$ is given by (13). Consistently with the neglect of surface tension in $(17)$, in evaluating $\dot{F}_{5}$ the effects of the surface strain rate $\dot{\varepsilon}$ on products of the form $\gamma d s$ are neglected Cagain, see the Appendix for the full expression), and hence

$$
\dot{\mathrm{F}}_{\mathrm{s}}=-2 \int_{\Gamma_{\mathrm{s}}} \gamma_{\mathrm{s}} \mathrm{kg} \dot{\mathrm{N}} \mathrm{d}_{\mathrm{s}}+\left\{2 \mathrm{Y}_{\mathrm{s}} \cos \phi_{\mathrm{D}}-\mathrm{Y}_{\mathrm{b}}\right]_{\mathrm{o}} \dot{\mathrm{a}} .
$$


The integral here represents the effect of adding matter at the rate $\dot{t} \equiv$ si to the surface (of course, $\dot{t}$ will generally be negative along $r_{s}$ when cavity is growing); as in our earlier discussion, $-k \dot{t}$ ds $=-k g \hat{N}$ ds is the rate of change of an arc ds, at a place where the curvature is $k$, due to matter addition. The last term of eq. (18) arises because the integral only gives the rate of change of $F_{S}$ from matter addition (or removal) from the existing arc $\Gamma_{s}$ but, as illustrated by the inset diagram in fig. 1 , it does not include the new surface element created during growth. As shown in the diagran, in cavity growth by da into the grain boundary, two arcs of length da cos $\phi_{0}\left(\phi_{0}\right.$ is the angle between $r_{s}$ and the negative $x$-axis at the cavity tipj are added to the cavity surface and the grain boundary diminishes by da, thus leading to the last term of (18) for $\dot{F}_{s}$. The subscript "0" on this last terth means that the $Y^{\prime} s$ refer to values at the cavity tip.

When eqs. (17) and (19) are added together there results

$$
\begin{aligned}
\dot{\mathrm{F}}=\dot{\mathrm{F}}_{\mathrm{e}}+\dot{\mathrm{F}}_{\mathrm{s}}=\dot{\mathrm{W}}+2 \int_{\mathrm{T}_{\mathrm{s}}}\left(w-\gamma_{s} \mathrm{x}\right) \sin d s \\
+\int_{\mathrm{r}_{\mathrm{b}}}(w-\sigma) g \dot{\mathrm{N}} d s+\left(2 Y_{s} \cos \phi_{0}-Y_{b}\right)_{0} \dot{\mathrm{a}}
\end{aligned}
$$

and, as shown in the Appendix, this equation is correct generally, 1.e., when surface tensions and associated dependences of products $y$ ds on surface strains are present, but note there that if surface tension $\mathbf{T}_{\mathbf{s}}$ exists at the tip, the distribution of 0 must be regarded as including a concentrated tension $\tau_{s} \sin \phi_{0}$ at the tip in this formula and in eqs. $(23,24)$ to follow. As noted in the Appendix, however, there are grounds for arguing that $\tau_{s}$ and $\tau_{b}$ must vanish at the tip. If we now recall 
the definitions of $\psi$ in eqs, (4) and (6), recognizing that $\kappa_{1}=*$, $\kappa_{2}=0$ in the present case, and then compare eqs. (14) and (19), we conclude that

$$
\left(2 Y_{s} \cos \phi_{0}-Y_{b}\right)_{0}=0 \text {, or } \cos \phi_{0}=\left(Y_{b} / 2 Y_{s}\right)_{0}
$$

during cavity growth. This is the well-known expression for the equilibriun angle $\phi_{0}$ at the apex of a grain boundary roid. The same expression has generaliy been assumed to apply during growth, and the present analysis provides a rationalization for this.

On the other hand, eq. (2), and hence eq. (14), were assuned to apply as equalities during growth. If instead they were assuned to apply as inequalities in the forth (notation of eq. (2))

$$
\dot{W} \geqslant \dot{F}-\int_{A} \mu \dot{N} d A,
$$

to allow for some forta of entropy production at the cavity tip in addition to the diffusive entropy production $(-J$ a $/$ /as $)$ already exhibited, o.g., in eq. (16), then combination of eq. (14), with $=$ replaced by $\geqslant$, and eq. (19) (which remains valid as an equality) leads to

$$
\left(2 \Upsilon_{s} \cos \phi_{0}-\gamma_{b}\right\}_{0} \dot{q} \leqslant 0
$$

This inequality will be satisfied during cavity growth (i.e., when $\dot{a}>0$ ) only if $\cos \phi_{0} \leqslant\left(\gamma_{\mathrm{b}} / 2 \gamma_{\mathrm{s}}\right)_{0}$; that is, only if $\phi_{0}$ is greater than or equal to t'le angle given by the equilibriun expression. During cavity shrinkage (i.e., sintering) it will be satisfied only if $\phi_{0}$ is less than or equal to the result of the equilibrium expression. Consequences of the inequality version of the Second law of Thermodynamics are perhaps not often surprising 
but this seems to be an exception. One might well expect that because the cavity advances by the drawing-in of material to the grain boundary at the cavity apex, under action of applied stress, that $\phi_{0}$ might decrease from the value given by the equilibrium expression as $\dot{a}$ increases from zero, and conversely. But thermodynamics prohibits this. If there is to be any deviation from the equilibrium expression, it must be in the opposite sense. In the circunstances it seens ressonable to sssume that o always retains the value given by the relation for equilibrium and, indeed, as shown by eq. (20) this must necessarily be the case if the cavity apex does not constitute a "point source" of entropy production. We have been careful to use the phrasing "angle given by the equilibrium expression" rather than "equilibriun angle" in this discussion. For example, owing to the dependence of the $r$ 's on surface strain it is possible that the angle $\phi_{0}$ given by the equilibriun expression in a stressed solid would differ from the corresponding angle $\Phi_{0}$ in an unstressed solid.

As a final expression for the energy release rate; which is perhaps most reyealing in comparing different nodels, we may rearrange eq. (17), which retains validity regardless of whether eq. (21) is regarded as an equality or inequality, to read

$$
\dot{W}-\dot{F}_{e}=\left[2 \int_{\Gamma_{s}} w(-\Omega \dot{N}) d s=\int_{\Gamma_{b}} w \Omega \dot{N} d s\right]+\int_{\Gamma_{b}} \sigma \Omega \dot{N} d s \cdot
$$

(Since eq. (17) neglects surface tension effects, so also does this equation. However, we show in the Appendix that this equation and, hence, our subsequent discussion in this section remains valid if $F_{e}$ is replaced by a new quantity $F_{e}$ which is, in fact, a more loglcal quantity to use in calculating an energy release rate, as $\dot{W}_{-} \dot{\mathrm{f}}_{\mathrm{E}}^{\prime}$, when surface tension 
tension effects exist. Also, as noted already, o then includes the concentrated tension $\tau_{s}$ sin $\phi_{0}$ at the tip). Equation (23) can be interpreted relative to fig. 2 where, in growing the void by some infinitesimal increment $\Delta a$, material of local thickness $\Delta n$ is removed from the notch surface and the grain boundary effectively thickens by an anount $\Delta \delta$. Hence

$$
\Delta W-\Delta F_{e}=2 \int_{\Gamma_{s}} w \Delta n d s-\int_{\Gamma_{b}} w \Delta \delta d x+\int_{\Gamma_{b}} \sigma \Delta \delta d x,
$$

and from this the difference between the Griffith-1ike model envisioned by Stevens and Dutton (1971) and the misfit model is clear. In the former case there is assumed to be no or negligible opening $\Delta \delta$ ahead of the crack, and all of the energy release goes to the first term of (24), repre. senzing strain energy of material diffused away fron the highly stressed notch surface. As remarked, it renains to be denonstrated that a model of this type will actually lead to cavity growth. On the other hand, in the Hull-Rinmer model and its generalizations, high stresses are alleviated by the misfitting material which effectively thickens the grain boundary at the notch tip. As we have denonstrated, in this case the $w$ terms are negligible and all of the energy release goes to the last term of (24), Which represents work done by local stresses in opening the grain boundary to accomodate the diffused natter.

It is tempting to regard the left side of (24) as being $G$ a where $G=\left(1-v^{2}\right) X^{2} / E$ is the usual energy release rate of linear elastic fracture mechanics. However, this is valid only in special cases, considered by Chuang (1974), of essentially steady, state growth in which the creep 
cavity is indeed thin (i.e., crack-like) in shape, and in which all effective thickening of the grain boundary is limited to a small vicinity of the void tip, with the diffused natter being accommodated by elastic distortion of the adjoining grains. In such cases Chuang shows, by developing numerically an explicit solution to the coupled (Iinearized) equations of elasticity and diffusion, that the $G$ level to drive the void is always greater than the Griffith level of eq, (11). Indeed, this is required by general considerations embodied in eq. (16) (in which we nay set $\dot{\mathbf{F}}_{s}=\left(2 \gamma_{s}-\gamma_{b}\right) \dot{a}$ for the present case $)$, since $(-J \partial u / \partial s)$ is necessarily positive. In fact, Chuang's analysis suggests that $K$ can never fall below approximately 1.7 times the Griffith value, and by an application of the J-integral (Rice, 1968a,b; Eshelby, 1970) he was able to derive an equation analogous to (24), negiecting the $w$ terms, and denonstrate that his munerieal rosults for $o(x)$ and $\delta(x)$ closely satisfied this equation. 
IV. TOTAL ENERGY CHANGE DUE TO INTRODUCING A CAVITY

Stevens and Dutton (1971) have considered the total energy change $\Delta \mathrm{E}$ due to introducing a cavity into a stressed body. In our notation E $=F$ - W where the external loads are assuned to be fixed so that W varies in proportion to the displacement of the loaded boundary. Following their classification, with slight nodifications, $\triangle E$ includes contributions from the following four sources (here we neglect conpletely surface tension effects]:

(1) change of energy due to void surface area introduced and lost grain boundary area $\left(\Delta \mathrm{F}_{5}\right)$,

(2) elastic strain energy change ( $\Delta F_{f}$ ) due to removal of baterial fron the body to form a void,

(3) work done by external loads ( $\Delta \overline{\bar{W}}$ ) during renoval of material,

(4) work done in processes of local matter rearrangenent, such as by local stress fields when the matter renoved fron the void is deposited at the g.b. ( $\triangle W^{*}$ ). (All other possible sources such as dislocation, slip, etc., are neglected).

It is important to note, as was not emphasized by Stevens and Dutton, that the $\Delta \bar{F}_{e}$ and $\Delta \bar{W}$ terns calculated in step (2) and (3) are not the total terms $\Delta F_{e}$ and $\Delta W$ (which, for example, enter into the energy release rate expression). Both $\Delta F_{e}$ and $\Delta W$ include a contribution from the work $W^{\prime}$ in step (4), and typically this is a very significant term. Indeed, the work $W^{\prime}$ is analogous to the effect of $\sigma$ term in eq. (6) for $u$ on a grain boundary; the part - $0 \Omega \delta N$ of $\mu \delta N$ in eq. (2) is essentially a work of type $w^{\prime}$, and we have seen its inportance in eq. (24). 
With the preceding classification of terms, the total energy change is

$$
\Delta E \equiv \Delta F_{s}+\Delta F_{e}-\Delta W=\Delta F_{s}+\Delta \bar{F}_{e}-\Delta \bar{W}+W^{\prime}
$$

In fact, the specific terms $\Delta \bar{F}_{e}, \Delta \bar{W}$ and $W^{\prime}$ depend on the sequence of operations and are different if natter (say, from a reservoir) is first inserted into the grain boundary, and then an equivalent amount of matter is removed from the body to form the void, and placed in the reservoir. Of course, the total effect, $\Delta F_{e}-\Delta W$, which these terms represent, is independent of sequence.

Consider a small elliptical cylindrical void lying along a g.b. as illustrated in fig. 3 with its najor axis normal to the direction of the uniaxial applied stress $\sigma_{m}$. We proceed to compute the energy change $\Delta E$ of this configuration in the following manner: First, we cut out the "volume" V (actually, an area in the 2-D model), thus creating a void on the boundary, and temporarily deposit it in an unstressed matter reservoir. This operation produces the free energy changes $\Delta F_{s}, \Delta \bar{F}_{e}$ and $\Delta \overline{\text { W }}$, where (Rice, 1968a)

$$
\Delta \vec{F}_{e}-\Delta \vec{w}=-\frac{\pi\left(1-v^{2}\right) \sigma_{\infty}^{2} a^{2}}{\vec{E}}(1+d / 2 a)
$$

Next we take the volume $V$ out of the reservoir and insert it inta the g.b., thus adding a non-uniform layer of material of local thickness $\delta$, such that

$$
\int_{g \cdot b .} \delta(x) d x=V=\pi a d .
$$


To get the ' process. This includes the work done on the surrounding material, plus the work necessary to bring the strain energy of the material being inserted to the proper level. Let $\sigma^{a}(x)$ be the normal tensile stress of an Inglis-type stress distribution induced by $a_{a}$, and let $\sigma^{b}(x)$ be the final total stress that results after the matter $v$ has been stuffed into the g.b. To compute $w^{\prime}$, we first cut the g.b., hold it in place by applying $\sigma^{a}$, and quasi-statically alter these stresses until the cut has apened locally by $\delta(x)$ (at which point the g.b. stresses are $\sigma^{b}(x)$ ). W' is simply the (negative) work done on the surface of the cut during this operation and if we assume the material is linearly elastic, then

$$
w^{\prime}=-\frac{1}{2} \int_{g . b .}\left(\sigma^{a}+\sigma^{b}\right) \delta d x
$$

of course, the material $V$ that is inserted. into the opened cut must be stressed and thus there will be an additional part of $W^{\prime}$ related to the strain energy increase in the inserted volume $V$ at state (b), but this portion is insignificant when $\sigma^{b}$ is small conpared to $E$. Still, if $\sigma^{b}$ is of the order of $\sigma_{w}$ over the region where the matter is added, the term is of order $\pi\left(1-v^{2}\right) \sigma_{\infty}^{2}$ ad/2E, which is of the sane order as $\Delta \overline{\mathrm{F}}_{\mathrm{e}}-\Delta \overline{\mathrm{H}}$ of (26) unless $\mathrm{d} / \mathrm{a} \ll 1$.

There are two observations to make about $w^{\prime}$. First, it is usually far greater in magnitude than $\Delta \bar{F}_{e}-\Delta \bar{W}$, and hence cannot be neglected. Suppose, for example, that the material is added uniformly over the entire 
g.b., so that $\sigma^{b}=\sigma^{a}$ and both have average values of $\sigma_{\infty}$. Then

$$
\begin{aligned}
W^{\prime}=-\sigma_{\infty} V & =-\sigma_{-\infty} \pi a d \\
& =\left(\Delta \bar{F} e^{-\Delta \bar{W})\left(E /\left(1+v^{2}\right) \sigma_{\infty}\right)(d / a) /(1+d / 2 a)} .\right.
\end{aligned}
$$

and the factor multiplying $\left(\Delta \bar{F}_{\mathrm{e}}-\Delta \bar{W}\right)$ will be much greater than unity unless $d / a$ is the same order as $0 / E$. Second, there is no unique value for $W^{\prime}$; it depends on how the natter is inserted. For example, concentration of the matter along a limited segment of the g.b. could make $\sigma^{b}$ large and negative there, so much so that $W^{\prime}$ could become positive (and indefinitely large). Also, addition of matter just over the highly stressed portions of the g.b., so as to reduce $\sigma^{b}$ to negligible values there, would produce a different expression for $W^{\prime}$ than (29). Since W' is not unique, neither are the terms $\Delta F_{e}$ and $A H$ and, hence, neither is the net energy change $\Delta \mathrm{E}$. Unique values result only for a given $\delta(x)$. In this sense it is meaningless to talk about an energy release rate (except in the liniting case of steady state crack-like cavity growth discussed in the last section) unless it is understood that the distribution $\phi(x)$ is detemined by the coupled equations of elasticity and diffusion as part of the analysis. 


\section{DISCUSSION}

Dutton and Puls (1978) in a comment on the formulation presented by Charles (1976), equated the erack extension force to the elastic fracture aechanics strain energy release rate $G=\left(1-v^{2}\right) x^{2} / E$. As we have seen, the usual fracture mechanics expressions are valid only in special circumstances. Essentially, their arguments are based on a Gxiffith-like crack model and treat the strain energy term in (24) as dominant. We have pointed out, however, that when a crack is lacated in a g.b. rather than in a perfect satrix, the g.b. is capable of acconmodating the material being removed from the crack surface and thus relax the high stress concentration ahead of the crack tip. Hence the strain energy contribution appears to be minor in the energy release expression and the major portion cones fron the work done by the boundary stress $\sigma$ on the opening $\Delta \delta$ as it appears in the last term of (24). Indeed, Charles (1978) noted in his reply that he believes that the "pv" work of insertion of a diffusible species in a highly stressed volume doninates in the stress rupture process, although he did not provide a full analysis as we do here.

Speight, Beere and Roberts (1978) have recently presented an intergranular crack growth nodel based on the mechanisns discussed here. Rather than relying on a full solution of relevant equations, as by Chuang (1974) and Vitek (1978), they imposed an approximste distribution $\delta$ (see eq. (1) of Speight et al., 1978) in arder to solve for the maximum stress in the g.b. Further, they attributed the energy release rate to the "plastic work" done by $\sigma_{\infty}$ on the crack volume $\left(w '=-\sigma_{\infty} v\right)$ as in eq. (29). But we have shown that the work term $w^{\prime}$ is dependent on the 


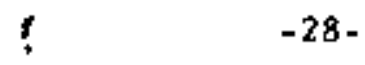

distribution of $\delta$ and their formulation does not appear to be fully consistent in this respect, although it is not clear as to how critically these assumptions affect their conclusions. 
VI. CONCLUSIONS

We have presented precise expressions for chenical potentials on grain boundaries and free surfaces, and noted that in representative cașes the strain energy contributions to these are negligible for the diffusive growth of grain boundary cavities. This is because surface diffusion tends to round-out a cavity nesr its tip, and grain boundary diffusion generates misfit stresses to alleviate its stress concentration, reducing local stresses to the order of $Y_{s} / D_{0}$. An evaluation of expressions for the energy release rate in diffusive cavity growth shows that the major energy sink is provided by the work of normal stresses on the grain boundary, as it is effectively thickened by matter addition, and not from the loss of strain energy of material diffused from the cavity tip. We discuss the computation of net energy changes associated with introduction of cavities, and point out that the dominant term is dependent on details of the matter placement along the grain boundary. In addition, we have commented on restrictions placed by thermodynamics on the angle formed by cavity walls where they join the grain boundary. 
VII. APPENDIX: SURFACE TENSION EFFECTS

At various points throughout the paper surface tension (or surface stress) effects were ignored for ease of presentation, with the promise that all would be made well here. Let $\theta^{l}, \theta^{2}$ be curvilinear coordinates in a surface (cavity surface, grain boundary) in some arbitrary reference configuration, and let $\mathrm{g}_{\alpha \beta}^{\circ}$ be the surface metric tensor $(\alpha, \beta=1,2)$ in this configuration. If the coordinate lines are regarded as being scribed onto the surface, in an elastic defornation the coordinates are convected and the metric tensor changes to $g_{a B}$. The (covariant) components of strain are defined as

$$
\varepsilon_{\alpha \beta}=\left(g_{\alpha \beta}-g_{\alpha \beta}^{0}\right) / 2
$$

The contravariant components (on the convected coordinates) $\tau^{\text {as }}$ of the surface tension tensor are defined such that $\tau^{a B}{ }_{b \varepsilon}{ }_{\alpha \beta} d A$ is the work of incremental elastic distortion $s e_{a B}$ of an area dA. Since $Y d A$ is the surface energy,

$$
\tau^{\alpha \beta_{\delta \varepsilon}}{ }_{\alpha \beta} \mathrm{dA}=\delta(\gamma \mathrm{dA})=\delta \gamma \mathrm{dA}+\gamma^{\alpha \beta_{\delta \varepsilon_{\alpha B}} \mathrm{dA}} .
$$

where $g^{a \beta}$ are the contravariant conponents of the metric, and we obtain the well-known relation between surface tension and surface energy (e.g., Herring, 1951,1952; Murdoch, 1976)

$$
\tau^{\alpha \beta}=\frac{\partial \gamma}{\partial \varepsilon_{\alpha \beta}}+\gamma g^{\alpha \beta},
$$


valid for arbitrary deformation. Note that if we define $\hat{\gamma}$ by $\hat{Y} d A_{0}=Y d A$, where $d A_{0}$ is the srea of the considered element in the reference configuration, then

$$
\tau^{\alpha \beta}=\frac{d A}{d A} \frac{\partial \hat{y}}{\partial \hat{c}} .
$$

IFon readers unfamilian with curvifinear tensons: If the coordinates lines are locally orthogonal at the point considered, and normalized so that $d \theta^{l}, d \theta^{2}$ are equal to the associated changes in arc length along the surface, then $g^{a 8}$ of eqs. $(A-2)$ and $(A-3)$ is just the Kronecker tensor and $\tau^{\alpha \theta}$ is the force acting in the $B$ direction per unit length of a cut along the surface with outward norral in the a direction. Also, when the reference configuration differs only by an infinitesimal anount from the current configuration, $\varepsilon_{\alpha \beta}$ is just the classical strain tensor).

Mechanical equilibriun equations for $\tau^{\alpha B}$ are complicated (Gurtin and Murdoch, 1975), but it is known that the field equations of equilibrium are fully inplied by the principle of virtual work, and the latter is most convenient for our purposes. Let A denote a region of cavity surface with bounding contour $C$. Iet $n_{i}$ be components (in 3-D space) of the outward normal to A [i.e., pointing away from the adjacent naterial, let $\sigma^{i j}$ be the stress tensor acting innediately beneath the surface (Latin indices $1, j$ have the range 1 to 3 ), and let $\tau^{\alpha}$ be components of the surface tension acting normal to $\mathrm{C}$. Hence the surface may be considered as a nembrane loaded with forces per unit area $-n_{2} \sigma^{i j}$ on $A$ and forces per unit length $r^{\alpha}$ along $C$. The principle of virtual work then requires that 


$$
\int_{A} \tau^{\alpha \beta} \delta E_{\alpha \beta} d A=\int_{C} T^{\alpha} \delta u_{\alpha} d C-\int_{A} n_{i} \sigma^{i j} \delta u_{j} d A
$$

for arbitrary virtual displacenent fields $\hat{\delta} u_{i}$ and associated virtual surface strains $\delta \varepsilon_{\text {as }}$. Some well-known consequences sre, for example, that

$$
T^{\beta}=m_{a} \tau^{\alpha \beta}, \tau^{\alpha \beta_{k B}}=n_{i} \sigma^{i j} n_{j}
$$

where $m_{a}$ denotes the outward normal (in the tangent plane of the surface) to $C$, and $K_{a b}$ is the surface curvature tensor.

An equation identical to (A-4) can be written for an arbitrary area A of the grain boundary, where $\tau^{\alpha \beta}$ and $T^{\alpha}$ are tensions in the $g . b$. , but now the integral involving $\sigma^{i j}$ nust be carried out over both sides of the grain boundary. Observe that when the grain boundary is flat, as considered here, the normal components of $o^{i j}$ are equal on both sides (and identical to what we have called $\sigma$ ). This means that for grain boundaries we can write $n_{i} \sigma^{i j} \delta u_{j}$ as $n_{i} \sigma^{i a_{0} u_{a}}$ where $\delta u_{G}$ are components of $\$ u_{i}$ parallel to the boundary and $\sigma^{i a}$ are shear stresses in the innediately adjacent material. Observe that for symmetrically loaded boundaries as in figs. 1 to $3,0^{i a}$ will reverse sign on crossing the boundary, and $o^{i a}$ is generally non-zero whenever the surface tensions in the boundary are non-uniform.

To calculate 4 (section II) we proceeded by calculating $6 \mathrm{~F}$ when a layer of local thickness $\delta t$ was added to cavity surfaces and/or grain boundaries, under conditions for which $\delta W=0$. We write $\delta F=\delta F_{e}+\delta F_{s}$, and observe that when matter is added, there are associated dispiacements 
$\delta u_{j}$ and strains $\delta \varepsilon_{a B}$ along $A_{s}$ (the cavity surfaces) and $A_{b}$ (the grain boundaries). The change in elastic strain energy is

$$
\begin{aligned}
\delta f_{e}=\int_{A_{s}} w \delta t d A & +\int_{A_{b}} w \delta d A-\int_{A_{b}} \sigma d t d A \\
& +\int_{A_{S}} \pi_{i} \sigma^{i j j_{j} d A}+\int_{A_{b}} n_{i} \sigma^{i t} \delta u_{a} d A
\end{aligned}
$$

The first three terms have been discussed in the body of the paper, the fourth corresponds to iten (iv) in the list enwmerated shortly before eq. (3)., and the last three terms together give the change in strain energy of the material lying outside the added layers. The last integral on $A_{b}$ covers both sides of the $g . b . ' s$ and $i t$ is noted that the normal stresses $\sigma$ and shear stresses $\sigma^{i \alpha}$ appear separately. Further, for symmetrically loaded grain boundaries as we consider, $\delta u_{\alpha}$ is the same on both sides of the boundary. The change in $F_{S}$ is

$$
\begin{gathered}
\delta F_{s}=-\int_{A_{s}} \gamma_{s}\left(\kappa_{1}+\kappa_{2}\right) t t d A+\int_{A_{s}}\left(\delta \gamma_{s}+\gamma_{s} g^{a B} \delta \varepsilon_{a B}\right) d A \\
+\int_{A_{b}}\left(\delta \gamma_{b}+\gamma_{b} g^{a B} \varepsilon_{a B}\right) d A .
\end{gathered}
$$

The first term has been discussed in the body of the paper. The second two are contributions, of type (iii) in the list shortly before eq. (3), due to effects of elastic strain in changing $\gamma$ by $\delta \gamma$ and $\mathrm{dA}$ by $g^{a B_{\varepsilon}} \varepsilon_{a B} d A$. Using eq. $(A-2)$, 


$$
\begin{aligned}
\delta F_{S}=-\int_{A_{S}} Y_{S}\left(\kappa_{1}+\kappa_{2}\right) \delta t d A & +\int_{A_{S}} \tau^{\Delta B} \delta E_{\alpha \beta} d A \\
& +\int_{A_{b}} \tau^{a B} \delta \varepsilon_{\alpha \beta} d A .
\end{aligned}
$$

Nechanical equilibrium is expressed by the principle of virtual work in eq. (A-4). Let us write this equation separately for the grain boundaries and for the cavities, taking $C$ to be the arc where the two make contact. Adding these equations together, we observe that contributions on $C$ cancel (because of mechanical equilibrium between $\tau_{s}$ and $\tau_{b}$ at the cavity tip, $2 \tau_{s} \cos \phi_{0}=\tau_{b}$ ) if there is a unique displacenent $\delta u_{j}$ at

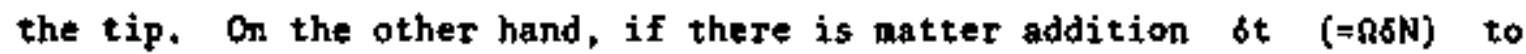
the grain boundary at the tip, so that the vertical components $t_{s} \sin \phi_{0}$ of $\tau_{s}$ separate by $\delta t$, the sum of the two terms involving $C$ is

$$
-\int_{C} \tau_{5} \sin \phi_{0} \delta t d C .
$$

Hence the resulting "grand" form of the principle of virtual work is

$$
\begin{aligned}
& \int_{A_{s}} \tau^{\alpha \beta} \delta \varepsilon_{\alpha \beta} d A+\int_{A_{b}} \tau^{\alpha \beta} \delta \varepsilon_{\alpha \beta} d A=\int_{C} \tau_{s} \sin \phi_{0} \delta t d C \\
& -\int_{A_{S}} n_{i} \sigma^{i j} \delta u_{j} d A-\int_{A_{b}} n_{i} \sigma^{i \alpha_{\delta}} \delta u_{\alpha} d A
\end{aligned}
$$

where. the last integral covers both sides of $A_{b}$. Since the actual field $\delta u_{i}, \delta \varepsilon_{\alpha \beta}$ associated with matter addition involves continuity of $\delta u_{\alpha}$ across $A_{b}$, it can be taken as the field to be entered in this form of 
the virtual work equation. Now, when we add together eqs, (A-6) and $(A-8)$, so as to write $\delta \mathrm{F}=\delta \mathrm{F}_{\mathrm{e}}+\delta \mathrm{F}_{\mathrm{s}}$, we find that because of eq. (A-9), all the terms involving $\tau^{a \beta}, \sigma^{i j}$ and $\sigma^{i \alpha}$ cancel one another. Hence

$$
\begin{gathered}
\delta F=\delta F_{e}+\delta F_{s}=\int_{A_{s}}\left[w-\gamma_{s}\left(\kappa_{1}+\kappa_{2}\right)\right] \delta t d A \\
+\int_{A_{b}}(w-\sigma) \delta t d A
\end{gathered}
$$

where here, and in eq. $(A-15)$ to follow, for simplicity in the presentation of formulae, the integral involving $a$ is understood to include a "delta function" contribution to $a$ at the cavity tip due to the vertical component of concentrated tension, $\tau_{s}$ sinto $\phi_{0}$ acting there.

Thus, if we write $\delta t=\Omega \$ \mathrm{~N}$ and observe that $\mu$ is defined by

$$
\delta F=\int_{A_{s}} \mu \delta N d A+\int_{A_{b}} \mu \delta N d A
$$

(in the case considered of matter addition under conditions for which 6w=0), then we obtain at once eqs, (4) and (6) in the body of the paper for $u$. Thus we see that while the terms associated with $\delta u_{i}$ and $\delta \varepsilon_{\alpha \beta}$ make first order contributions to $\delta F_{e}$ and $\delta F_{s}$ separately, they make only higher order contributions to the total $\delta F$. There is, however, a curious result at the tip. Because $\sigma$ has the delta-function contribution there, of integrated intensity $\tau_{s} \sin \phi_{0}$, we must conclude that $\mu$ has a similar negatively infinite delta-function form of integrated intensity $-\tau_{5} \sin \phi_{0} \Omega$, at the tip. We leave open the interpretation to be given to this. Certainly, to the extent that thermodynamics required continuity 
of $u$, such a condition can be met only if the material adjusts its state of surface strain at the tip in a manner such that $\tau_{s}$ (and hence $\tau_{b}$ too) vanishes there.

In the discussion of plane strain growth of a cylindrical cavity, surface tension effects were ignored in deriving eqs. (17) and (18). Now we can drop indices on $\tau^{\alpha \beta}$ and $\varepsilon_{\alpha \beta}$, Hriting $\tau$ and $\varepsilon$ for the components in the plane of deformation. With the previous discussion as background, the full version of eq. (17) is readily sees to be

$$
\begin{gathered}
\dot{\mathrm{F}}_{e}=2 \int_{\Gamma_{s}} w \dot{\mathrm{N}} \mathrm{ds}+\int_{\Gamma_{b}} w \dot{N} d s+\dot{W}-\int_{\Gamma_{b}} \sigma \Omega N d s \\
+2 \int_{\Gamma_{s}} n_{i} \sigma^{i j_{\dot{u}_{j}}} d s+\int_{\Gamma_{b}} n_{i} \sigma^{i x_{u_{x}}} d s
\end{gathered}
$$

where the integral on $r_{b}$ covers both sides of the g.b. and, as in figs. 1 to 3 , the $x$ direction is parallel to the grain boundary. Here $1, j$ range over $x, y$. Similarly, the full version of eq. (18) is:

$$
\begin{gathered}
\dot{F}_{s}=-2 \int_{T_{s}} Y_{s} k \sin d s+\left(2 Y_{s} \cos \phi_{0}-Y_{b}\right)_{o} \dot{a} \\
+2 \int_{T_{s}} \tau \dot{\varepsilon} d s+\int_{T_{b}} \tau \dot{\varepsilon} d s .
\end{gathered}
$$

Again, a grand form of the principle of virtual work may be written, analogously to $(A-9)$, and this is 


$$
\begin{aligned}
& 2 \int_{\Gamma_{s}} \tau \delta \varepsilon d s+\int_{\Gamma_{b}} \tau \delta \varepsilon d s \\
& \quad=-\left(\tau_{s} \sin \phi_{0} \delta t\right)_{0}-2 \int_{\Gamma_{s}} n_{i} \sigma^{i j_{\delta u_{j}} d s}-\int_{\Gamma_{b}} \pi_{i} \sigma^{i x_{\delta u_{x}} d s .}
\end{aligned}
$$

Thus, when eq. $(A-12)$ and $(A-13)$ are added together the terms multiplying $\dot{c}$ and $\dot{u}_{i}$ cancel, and we obtain eq. (19) in the body of the paper (which we remarked there to be valid in the general case when surface tension effects exist, so long as $\sigma$ is taken to include the concentrated tension at the tip--if, indeed, this is non-zero).

Finally, if eq. $(A-14)$ is used in $(A-12)$, and then $(A-12)$ is rearranged to the form of eq. (23) in the body of the paper, we obtain

$$
\dot{W}-\dot{\mathrm{F}}_{\mathrm{e}}^{*}=\left[2 \int_{\Gamma_{\mathrm{s}}} w(-\Omega \hat{N}) \mathrm{ds}-\int_{\Gamma_{\mathrm{b}}} w \dot{N} \mathrm{ds}\right]+\int_{\Gamma_{\mathrm{b}}} \sigma \Omega \dot{N} \mathrm{ds}
$$

(1. e., the same right side as $€ q .(23))$ if we define

$$
\dot{\mathrm{F}}_{e}=\hat{\mathrm{F}}_{\mathrm{e}}+2 \int_{T_{s}} \tau \dot{\varepsilon} \mathrm{ds}+\int_{r_{b}} \tau \dot{\varepsilon} d s .
$$

When we recall that $\dot{F}_{\theta}$ can be written as the integral over the volune of the body of stress components times corresponding components of strain rate, these added terms seen certainly to be appropriate, and $\dot{W}-\dot{F}_{e}^{\prime}$ seens to be the obvious extension of the concept of an energy release rate to cases In which surface tensions exist during cavity growth. 
VIII. ACKNOWLEDGENENT

The work was supported by the U. S. Department of Energy under contract EY-76-S-02 30B4 A003 with Brown University. We are grateful for several discussions with Ors. R. Dutton and M. P. Puls of the Whiteshell Nuclear Research Establishment, Pinawa, Manitoba, Canada. In fact, Section IV of the paper and Figure 3 are based on a letter which we wrote to Dr. Dutton in 1974, in reply to sone comments by him on our work (Chuang and Rice, 1973). Continuing discussions with various individuals since that time have convinced us that the issues of that letter, and the more general perspective on diffusive cavity growth that we developed subsequently (forming the earlier portions and the Appendix of this paper) might be suitable for publication. 
REFERENCES

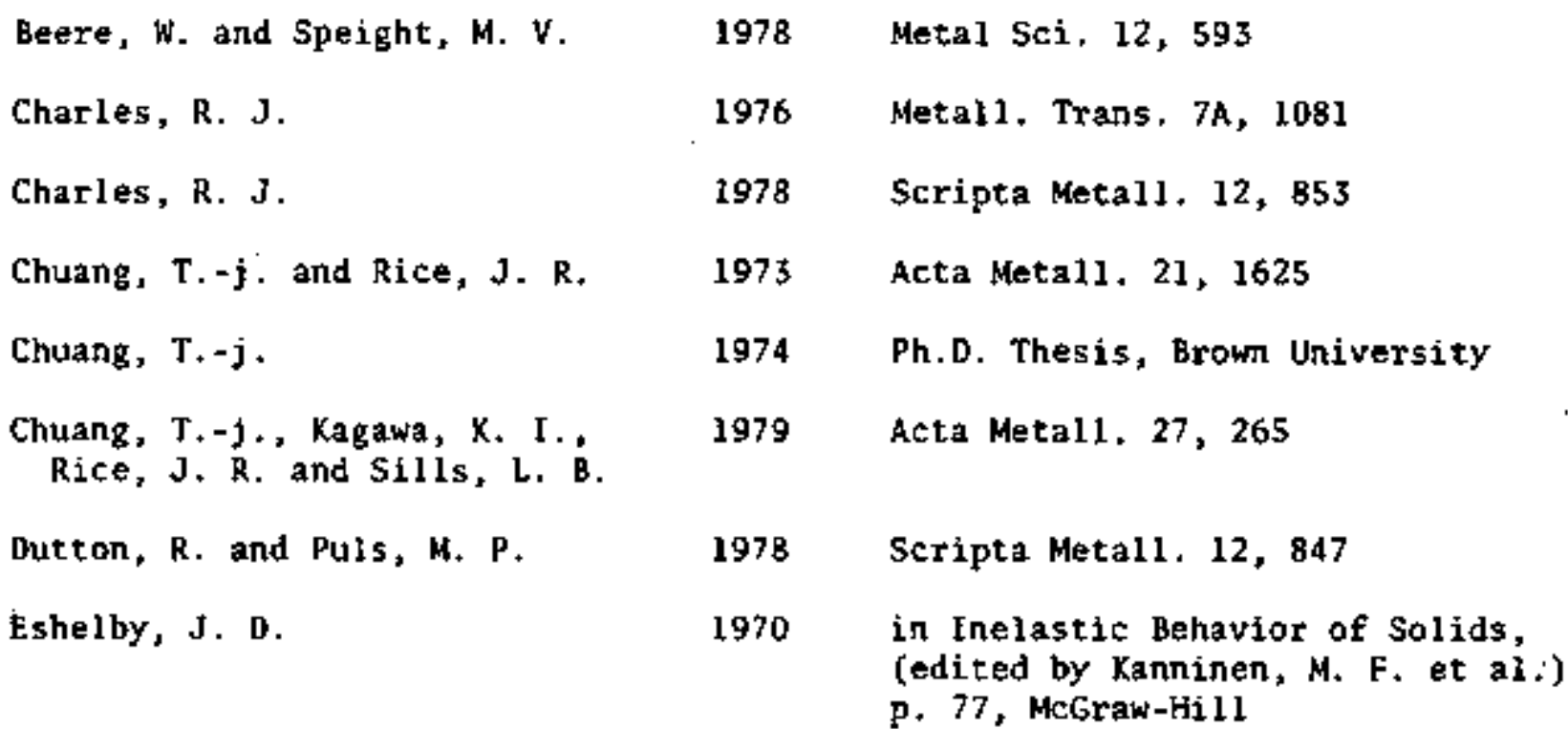

Gurtin, M. E. and Murdoch, A. I. 1975

Arch. Rat. Mech. Anal. 57, 291

Heald, P. T. and Speight, M. V.

1977

wat. Sei. Eng. 29, 271

Herring, $c$.

$19 \$ 1$

in The Physics of Powder Metallurgy, (edited by Kingston, W. E.) MeGrawHil1

Herring, C.

1953

in Structure and Properties of Solid Surfaces, (edited by Goner, R, and Snith, C. S.), p. 5, Univ. Chicago Press

Hu11, D, and Rimer, O. E.

1959 Phil, Nag. 4,673

McCartney, L. N.

1977 Acta Metall, 25, 221

Murdoch, A. I .

1976

Q. J. Mech. Appl. Math 29, 245

Raj, R. and Ashby, M. F.

1975

Acta Metal1. 23, 653

Rice, J. R. and Drucker, D. C. 1967

Int, 1, Fract. Mech. 3, 19

Rice, J. R.

$1968 \mathrm{a}$

in Fracture, (edited by Liebowitz,

Rice, J. R.

$1968 b$

H. , vol. 2, p. 191, Acadennic Press

Rice, J.R.

1974

$J$, App 1, Nech, 35, 379

in The Constitutive Equations in Plasticity, (edited by Argon, A. S.) M.I.T. Press

Rice, J, R. 
$-40$

Speight, M. V. and Harris, J. E, 1967 Met. Sei. J. 1, 83

Speight, M. V., Beere, W. B., 1978 Mat. Sci. Eng. 36, 155 and Roberts, G.

Stevens, R. N, and Dutton, R. 1971 Mat. Sci. Eng. 8, 220

vitek, V.

1978 Acta Metal1, 26, 1345

Vitovec, F. F.

1972 J. Mat. Sci, 7,615

Weertman, $\mathrm{J}$.

1973 Scripta Metall. 7, 1129 
$-41-$

FIGURE CAPTIONS

Figure 1. Cylindrical void in a grain boundary.

Figure 2. Illustration of matter transfer in diffusive cavity growth.

In growth $\Delta a$ a layer of local thickness $\Delta \mathrm{n}$ is removed from the cavity surface and the grain boundary, effectively, thickens by a local amount $\Delta \delta$ due to matter addition.

Figure 3. An elliptical cavity in a linear elastic material; $\sigma^{a}$ is the grain boundary tensile stress if there is no matter placement in the grain boundary, and $\sigma^{b}$ results after placement. 


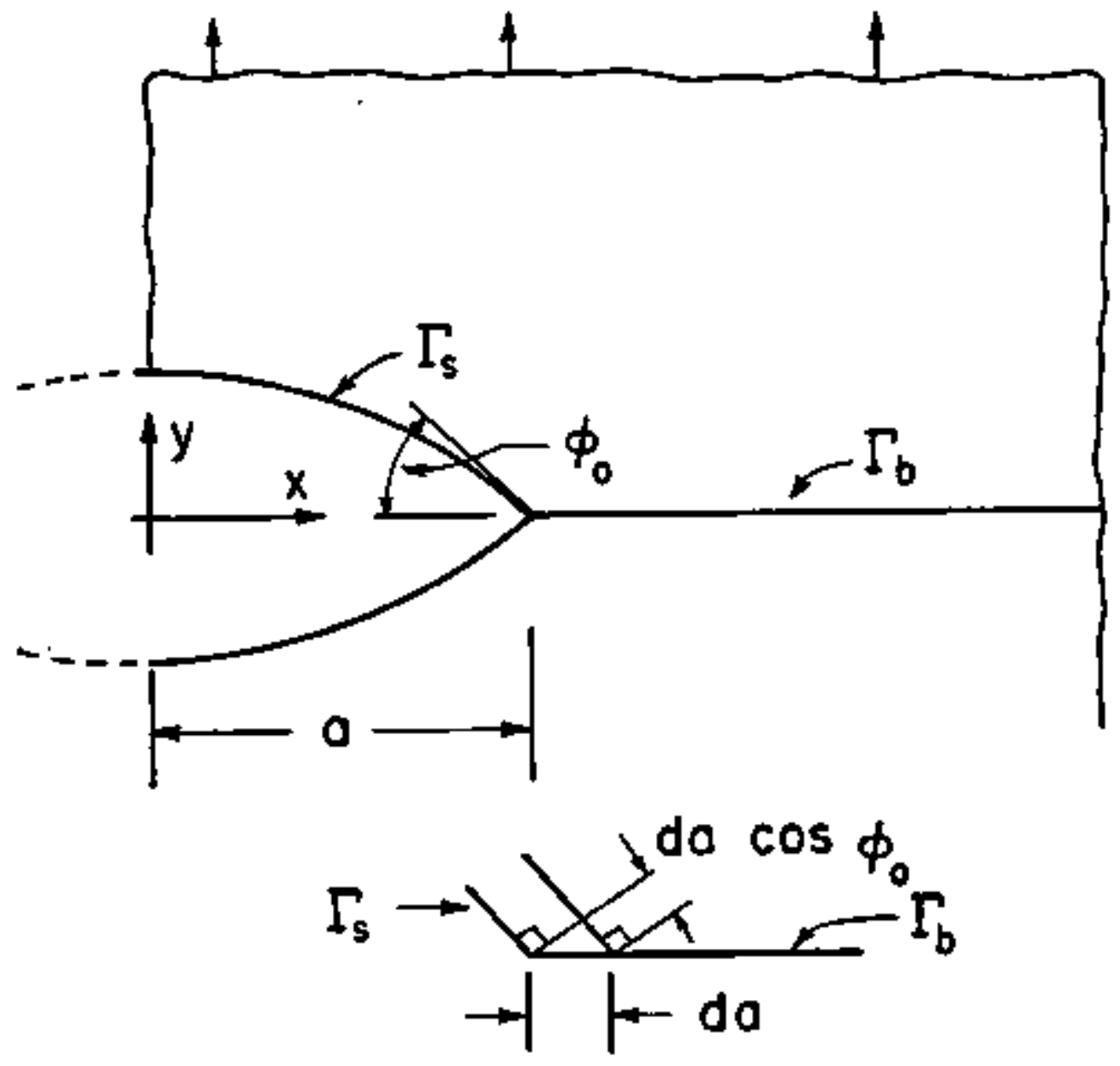

Figure 1 
$-43-$
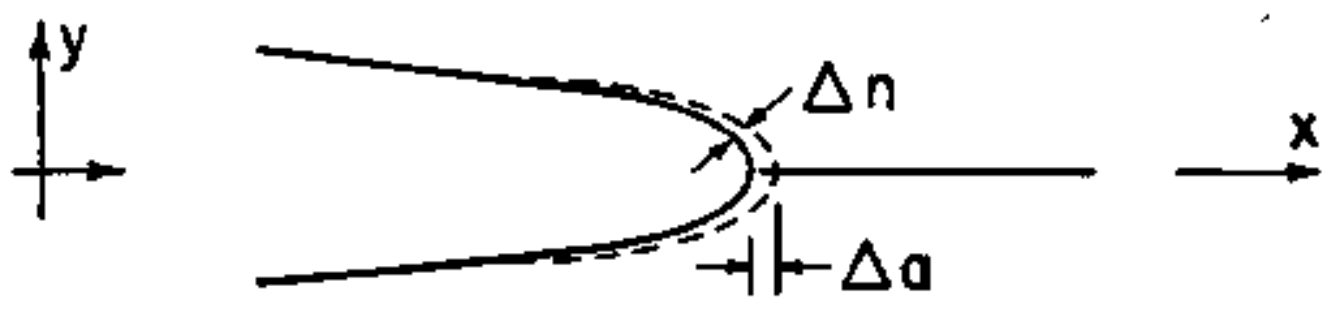

(a)
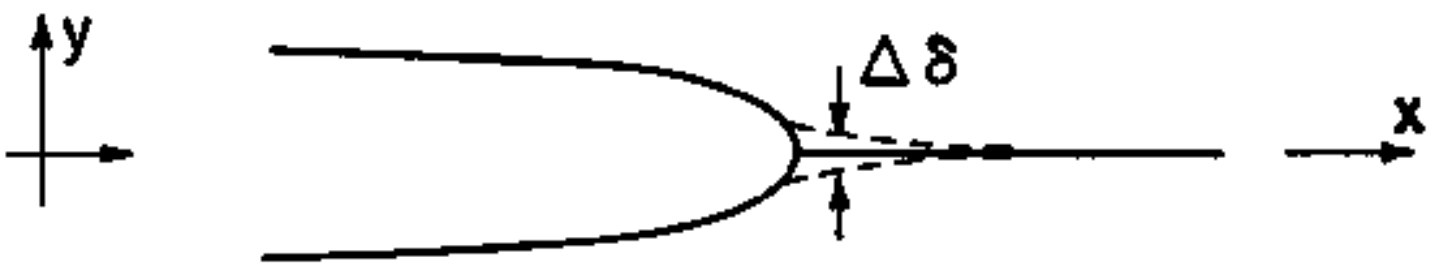

(b)

Figure 2 


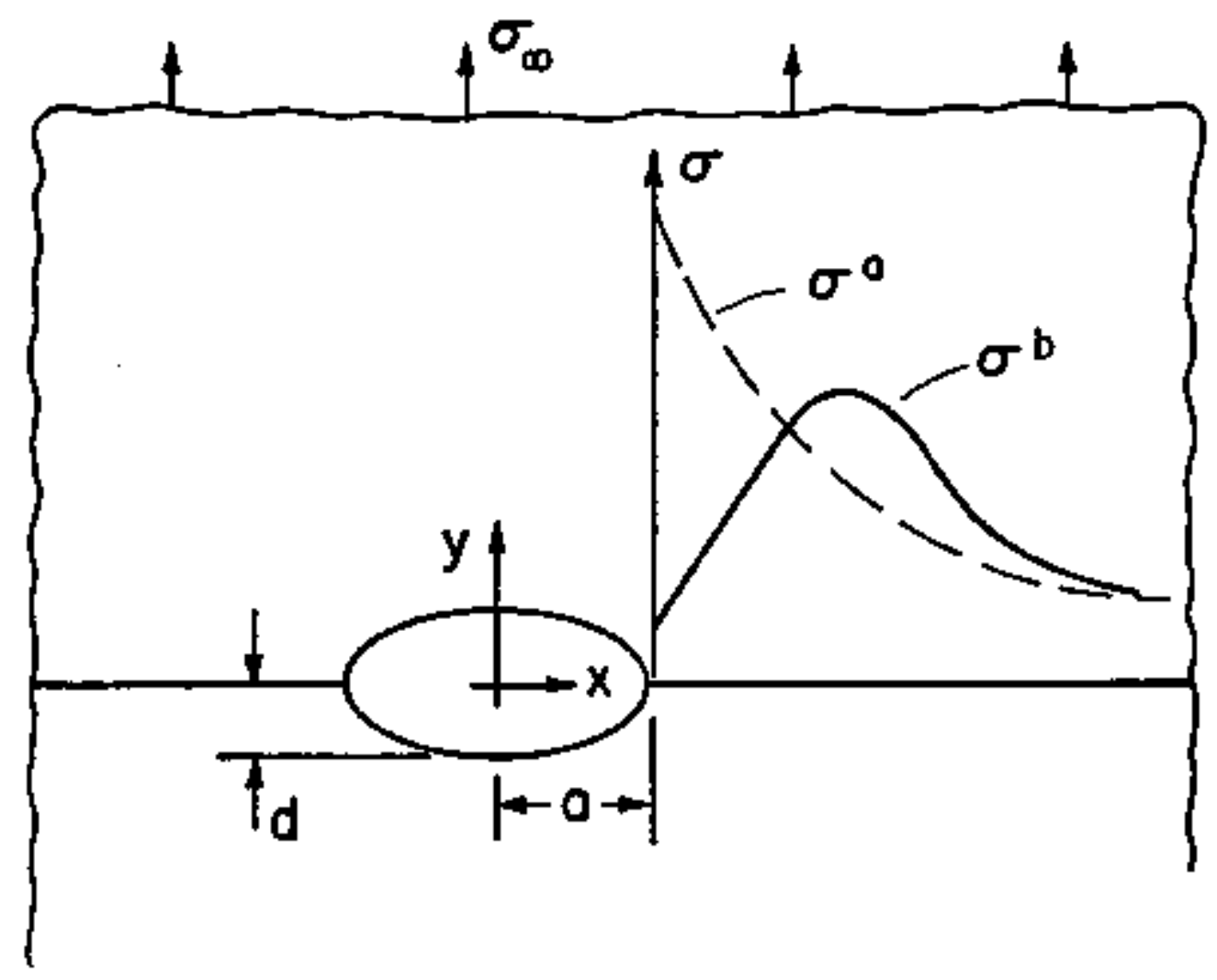

Figure 3 